\title{
A Novel Approach to 'Making Sense' Out of the Copenhagen Interpretation
}

\author{
Armin Nikkhah Shirazi \\ University of Michigan, Department of Physics, 450 Church Street, Ann Arbor MI 48109
}

\begin{abstract}
This paper presents a concise exposition of the Dimensional Theory, a novel framework which helps make sense out of the Copenhagen Interpretation as it explains the peculiarities of quantum mechanics in a way that is most consistent with that interpretation ${ }^{1}$.
\end{abstract}

Keywords: Copenhagen Interpretation, Dimensional theory, Actualizable, Areatime

PACS: $<03.65 . \mathrm{Ta}>$

\section{INTRODUCTION}

The Copenhagen interpretation, though the most widely accepted interpretation of quantum mechanics, does not seem to give a clear meaning to the theory's mathematical formalism. This paper will present a novel framework which helps make sense out of it by answering certain "why" questions the interpretation leaves unclear. For example, why does a quantum system not have definite properties prior to being measured? Why is there a "cut" between the classical observer and the quantum system? Why is there such a thing as "particle-wave duality"? The underlying framework that will be used to offer an answer to these questions is called the Dimensional Theory.

\section{ACTUAL VS. ACTUALIZABLE}

As this framework is new and unfamiliar, it may help to first present a motivational observation. When one attempts to represent an object in a higher-dimensional space, then aspects of that representation which depend on extent along dimensions in that space not associated with the object itself or an embedding surface must be represented such that the higher-dimensional representation includes all possible values along those dimensions. In short, such an object must be represented as a "superposition" of higherdimensional representations in a higher-dimensional space. For instance, a point $\left(x_{0}, y_{0}\right)$ in a 2-dimensional plane that is not embedded as a surface in 3-space must be represented as an infinitely long line, or, put more provocatively, as a "superposition" of an infinite number of points with common $x$ and $y$ coordinates but different $z$ coordinates. Furthermore, the superposition "collapses" if a third coordinate $z_{i}$ is attributed to the point $(x, y)$ to transform it into the point $\left(x, y, z_{i}\right)$ which now becomes a single point in

\footnotetext{
${ }^{1}$ A recording of the talk based on this material can be viewed at http://youtu.be/GurBISsM308
} 
3-space, as in fig. 1
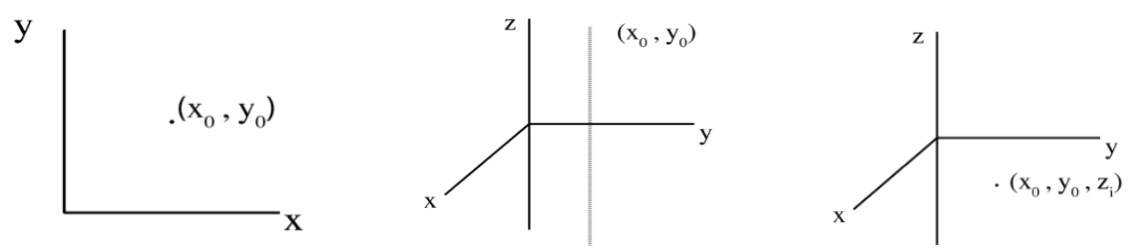

FIGURE 1. The point $\left(x_{0}, y_{0}\right)$ in $R^{2}$ represented in $R^{3}$ before and after "collapse"

Hence, there are two ways of constructing an infinitely long straight line in Euclidean 3-space: (1)Either integrate over all $z$-values at a certain value for $x$ and $y$, which in effect means the line is constructed by an infinite number of points, each specified by three coordinates, or (2) specify just two coordinates, and allow all $z$-values to be included in the representation "by default", as it were. To the extent that this difference in construction is contemplated at all, it appears that the resultant objects are currently taken to be equivalent. However, as table 1. shows, they are not, and to give a label to this distinction, we will call the line as constructed the first way an actual line, and as constructed the second way an actualizable line.

TABLE 1. Differences in properties between the actual and actualizable line in Euclidean Space

\begin{tabular}{|c|c|c|}
\hline & Actual & Actualizable \\
\hline $\begin{array}{l}\text { Applicable Metric Interval } \\
\text { Locally Displaceable }\end{array}$ & $\delta_{i j} d x^{i} d x^{j} \quad i, j=1,2,3$ & $\delta_{i j} d x^{i} d x^{j} \quad i, j=1,2$ \\
\hline Metric Relations between perpendicular lines & Directly defined & Not directly defined \\
\hline Effect of addition of third coordinate information & No collapse & Collapse \\
\hline
\end{tabular}

The most salient distinction between actual and actualizable objects is that whereas the former, as objects in the space in which they are represented, never exist in a superposition, the latter, as manifestations of actual objects in a lower-dimensional space, always exist in a superposition. The apparent absence of any discussion of these differences in the mathematical literature may indicate that this could possibly be an overlooked area of mathematics with high relevance to physics.

\section{THE DIMENSIONAL THEORY}

The Dimensional Theory (DT) is a simple framework which applies the ideas discussed above to the geometry of spacetime to derive the free-particle Feynman path integral from a few axioms, in the process explaining the peculiarities of quantum mechanics in a way that is most consistent with the Copenhagen Interpretation.

Let us first present a conceptual discussion. The DT assumes that there exists a limit, symbolized by $\left|U_{3 \max }\right|$, in which any region of spacetime $V \tau$ reduces to a constant quantity of variable shape of a 1-dimension reduced analog called areatime. The metric interval associated with objects in areatime is naturally distinct from the spacetime metric interval, and consequently so is the associated proper time dimension. Since for spacetime objects, a definite proper time is required to form the 4-dimensional object we call a world line, areatime objects with their distinct proper time dimensions lack world 
lines in spacetime. By an axiom, it is assumed that areatime objects can be represented in spacetime in terms of a "superposition" of all possible world lines, but that these are actualizable. In particular, this means that they "collapse" once a spacetime object with an actual world line emerges out of the areatime object. Another axiom stipulates that the passage of time for the areatime object is compared with, or "translated into", passage of time along any actualizable world line via a mechanism which will be called the angular dual bilateral symmetry. The construction of the simple symmetry is as follows:

1. The proper time $\tau_{A}$ of the areatime object and $\tau$ of each actualizable worldline must be represented along orthogonal directions in an abstract plane because we know from the phenomenon of time dilation that non-orthogonal time dimensions cannot be considered distinct: we consider time-dilated objects to still exist in spacetime.

2. The passage of time is mapped from $\tau_{a}$ to $\tau$ indirectly via the rotation of an amplitude $\tau_{r} \equiv \sqrt{\tau^{2}+\tau_{A}^{2}}$ with period $T \propto \tau_{A}$

3. Rotations of the amplitude in forward and backward directions are "superimposed" so that the resultant transformation is just the identity (due to the cancellation of the opposite rotations). This leads to two new symmetries not found in an ordinary rotation: invariance under angular displacement for any angle (as opposed to just multiples of $2 \pi$ ) and under reflection. Hence the name for this symmetry.

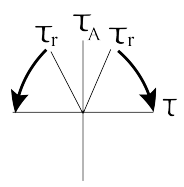

FIGURE 2. The symmetry: Two amplitudes $\tau_{r}$ rotate in opposite directions in the plane of $\tau$ and $\tau_{A}$

The symmetry ensures that over any finite amount of time the outcome of the transformation is no change (i.e. the identity) while the process allows a comparison of distinct time dimensions. Metaphorically, it is a clock that goes both forwards and back and thereby remains frozen in time. Again, the point of this is to provide a mechanism for comparing the passage of time without a net transformation between two distinct proper time dimensions, which preserves their orthogonality, required to maintain distinctness. By assumption, the endpoints of $\tau_{A}$ which coincide with the boundary of $\left|U_{3 \max }\right|$ are identified, resulting in the manifestation of periodicity of $\tau_{A}$ to spacetime observers. Because $\tau_{A}$ only exists in the small limit in which spacetime vanishes, it may repeat itself many times as it is compared against consecutive intervals along $\tau$, as depicted in fig. 3

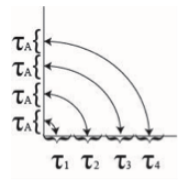

FIGURE 3. The same interval $\tau_{A}$ is compared to consecutive distinct intervals along $\tau$

This turns the abstract plane into a Riemann surface and implies that $\tau_{A}$ has to be modeled as an imaginary period: $\tau_{A}=i T / 2 \pi$. If the angle associated with the amplitude is $\varphi \equiv \tan ^{-1}\left(\tau_{A} / i \tau\right)$ (where $\tau_{A}$ is divided by $i$ to make the argument real), then a simple reparameterization $\theta \equiv \cot \varphi=i \tau / \tau_{A}$ turns the symmetry into two complex conjugate 
phases of the form $e^{\frac{\tau}{\tau_{A}}}$ where the normalization of $\tau_{r}$ will be absorbed in the normalization of the path integral at the end. We now present an abbreviated mathematical derivation of the path integral of a single free particle:

- Axiom I: $\lim _{V \rightarrow 0} V \tau=A \tau_{A} \equiv\left|U_{3 \max }\right|$ (where $A$ is the area associated with the limit)

- Axiom II: Objects which actually exist in areatime must be represented in spacetime by a superposition of all possible actualizable worldlines of the object(s) in spacetime into which they can emerge.

- Axiom III: The passage of time along the proper time of an areatime object is mapped to that along each actualizable worldline by means of the angular dual bilateral symmetry in such a way that the points on $\tau_{A}$ which coincide with the boundary of $\left|U_{3 \max }\right|$ are identified.

- Axiom IV: If the areatime object forms a "complex" with other areatime objects such that the limit $\left|U_{3 \max }\right|$ is exceeded, the actualizable mass associated with it transforms into actual mass.

Here, actualizable mass $m$ is always associated with a superposition of properties, but actual mass $\underline{m}$ (underlined for distinction) never is, which sharply separates the domains of classical and quantum physics. A fuller discussion of this distinction, including its impact on the equivalence principle, is given in reference [2]. Now, taking axiom I to apply to the "free particle", the object underlying it, actually existing in areatime, lacks a spacetime worldline. From axiom II follows that the particle manifestation must be modeled in terms of a superposition of all possible actualizable worldlines. We can think of each actualizable worldline as a path in space that is traversed over a time interval. This time interval is compared to the passage of the time for the underlying areatime object using the mechanism outlined in axiom III and elaborated above. For a single free particle, the appropriate substitution is $\tau_{A} \Rightarrow \pm \frac{i \hbar}{m c^{2}}$ where the \pm indicates that the symmetry results in both phase factors. Then we have

$$
e^{\frac{\tau}{\tau_{A}}} \Rightarrow e^{\frac{m c^{2}}{ \pm i \hbar} \tau}=e^{\mp i \frac{m c^{2}}{\hbar} \tau}=e^{\mp i \frac{m c}{\hbar} \int d s}=e^{ \pm i \frac{S}{\hbar}}
$$

Where $\int d s=s=c \tau$ is the finite spacetime interval and $S=-m c \int d s$ is the classical relativistic free-particle action. In the non-relativistic limit, we get

$$
e^{ \pm i \frac{S}{\hbar}}=e^{ \pm \frac{i}{\hbar} \int L(\mathbf{r}, \dot{\mathbf{r}}, t) d t} \sim e^{ \pm \frac{i}{\hbar} \int\left(\frac{1}{2} m v^{2}\right) d t}
$$

Where $L(\mathbf{r}, \dot{\mathbf{r}}, t)$ is the Lagrangian associated with the system. Under the DT then, this fundamentally dynamic quantity arises out of a geometric mechanism for comparing two distinct proper time dimensions. Since the comparison involves each actualizable worldline separately, both phase factors must be associated with each actualizable path, but in practice one associates only one of these with the integral over all paths.

Deriving the free particle path integral, written symbolically as $\int_{\mathbf{r}}^{\mathbf{r}^{\prime}} \mathscr{D} x(t) e^{i i^{S}}$, from this point on is well understood [1] and due to space constraints the explicit calculation will not be performed here. The end result in terms of the propagator $K\left(\mathbf{r}^{\prime}, \mathbf{r}, t\right)$ is

$$
K\left(\mathbf{r}^{\prime}, \mathbf{r}, t\right)=\left(\frac{m}{i 2 \pi \hbar\left(t_{f}-t_{i}\right)}\right)^{3 / 2} e^{\frac{i m}{2 \hbar}\left(\frac{\left(\mathbf{r}^{\prime}-\mathbf{r}\right)^{2}}{\left(t_{f}-t_{i}\right)}\right.}
$$


Again, this looks exactly like the standard expression but the path integral here is interpreted as an integral over all actualizable, not actual paths. The propagator is only associated with one of the phases but the fact that the transition probability requires taking its absolute square indicates that both complex conjugate phase factors due to $e^{\frac{\tau}{\tau_{A}}}$ contribute equally to the spacetime manifestation. The propagator allows the determination of a quantum state $\Psi\left(\mathbf{r}^{\prime}, t_{f}\right)$ from an earlier state $\Psi\left(\mathbf{r}, t_{i}\right) \equiv \Psi_{0}$ :

$$
\Psi\left(\mathbf{r}^{\prime}, t_{f}\right)=\int_{-\infty}^{\infty} K\left(\mathbf{r}^{\prime}, \mathbf{r}, t\right) \Psi_{0} d \mathbf{r}^{3}
$$

from which it follows that the quantum state $\Psi\left(\mathbf{r}^{\prime}, t_{f}\right)$ is also actualizable.

Axiom IV applies when a quantum system is 'measured': this represents the 'actualization' or emergence of an actual spacetime object: The detector-system complex in areatime exceeds the limit $\left|U_{3 \max }\right| \rightarrow$ actualizable mass $m$ is transformed to actual mass $\underline{m} \rightarrow$ The object is associated with a spacetime proper time $\rightarrow$ the superposition of actualizable worldlines "collapses" $\rightarrow$ An actual spacetime worldline begins to form. This is identified with the "wave function collapse", but the eigenstate $\underline{\psi}$ is now actual

$$
\Psi \stackrel{m \rightarrow \underline{m}}{\longrightarrow} \underline{\psi}
$$

For objects like electrons this is evidently reversible, as they soon "spread out" again, but sufficiently rapid successive "measurements" may forestall the "de-actualization".

Finally, since in this framework the wave function phase factor $e^{-i \frac{\hat{H} t}{\hbar}}$ ultimately comes from the factor $e^{\frac{\tau}{\tau_{A}}}$ via $e^{i \frac{S}{\hbar}}$, and proper time is proportional to the metric interval, the wave function encodes within its mathematical expression areatime metric information. In particular, if $\Psi$ describes entangled components, it models the spacetime manifestation of a system confined to a local region in areatime, parts of which can give rise to the emergence of actual objects in distant regions in spacetime with correlated properties.

\section{CONCLUSION}

This paper presented a novel framework, the Dimensional Theory, which permits one to make sense of the Copenhagen Interpretation of quantum mechanics. While unfamiliar, it makes a definite and highly unexpected prediction arising from the distinction between actual and actualizable mass: photons, as purely actualizable objects, do not produce gravity fields. This can at least in principle be tested against calculations in the literature, such as by Tolman et. al.[3], which assume that radiation in transit is a field source.

\section{REFERENCES}

1. R. P. Feynman and A. R. Hibbs, "Quantum Mechanics and Path Integrals" (Emended Edition), edited by D. F. Styer, Dover Publications, Mineola, (2010)

2. A. Nikkhah Shirazi "Are the Concepts of Mass in Quantum Theory and in General Relativity the Same?" available at Deep Blue, University of Michigan, http://hdl.handle.net/2027.42/87999 (2011)

3. R. C. Tolman, P. Ehrenfest, and B. Podolsky, "On the Gravitational Field Produced by Light", Physical Review, 37, 604-615 (1931) 